\title{
Variant of Takotsubo Cardiomyopathy with Co-Existent Severe Coronary Artery Disease - A Case Report
}

\author{
N Craig, S Krishnamoorthy*, M Halim and A Venkataraman \\ George Eliot Hospital NHS Trust, United Kingdom \\ *Corresponding author: S Krishnamoorthy, George Eliot Hospital NHS Trust, Nuneaton, CV10 7DJ, United Kingdom
}

\begin{abstract}
TakotSubo Cardio Myopathy (TSCM) is a reversible form of cardiomyopathy characterized by transient left ventricular (LV) systolic dysfunction. Clinically often mimics acute myocardial infarction (AMI) with chest pain or breathlessness with electrocardiographic, cardiac marker(s) and LV wall motion abnormalities. In a third of patients, significant emotional or physical stress precedes the presentation. Often, these patients have normal epicardial coronaries or non-flow obstructive coronary disease. In most patients, LV function shows complete recovery within weeks with favorable outcomes. We report a unique case of TSCM following sudden emotional stress with typical transient apical ballooning; nevertheless, with severe co-existent triple vessel coronary artery disease and complete recovery of cardiac function on medical management.
\end{abstract}

Keywords: Tako-Tsubo Cardiomyopathy; Apical Ballooning; Coronary Artery Disease

\section{Case Presentation}

A 73 year old hypertensive female admitted with sudden onset intermittent chest pains and shortness of breath $(<24$ hours after the death of her granddaughters in a road traffic accident). There were no other significant past medical history of note and she never smoked. On arrival, she was tachycardic (heart rate 102) in sinus rhythm, normotensive and clinical examination was unremarkable. Her initial ECG (Figure 1) demonstrated sinus tachycardia with deep $\mathrm{T}$ wave inversions in both precordial and limb leads. Her bloods revealed high sensitivity Troponin-T of 395ng/L [normal

reference range $<15 \mathrm{ng} / \mathrm{L}]$. Her bedside echocardiogram revealed typical pattern of "apical ballooning" with severe hypokinesis in the anterior, lateral, septal and inferior walls of the left ventricle, sparing the basal walls. Her left ventricular systolic function was moderately impaired with an ejection fraction (EF) of $40 \%$ (Figure 2). Both her clinical presentation and echocardiographic appearances were highly suggestive of "Takotsubo Cardiomyopathy". Nevertheless, she was conventionally treated for presumed "Non-ST elevation myocardial infarction (NSTEMI)" at the initial stages [1].

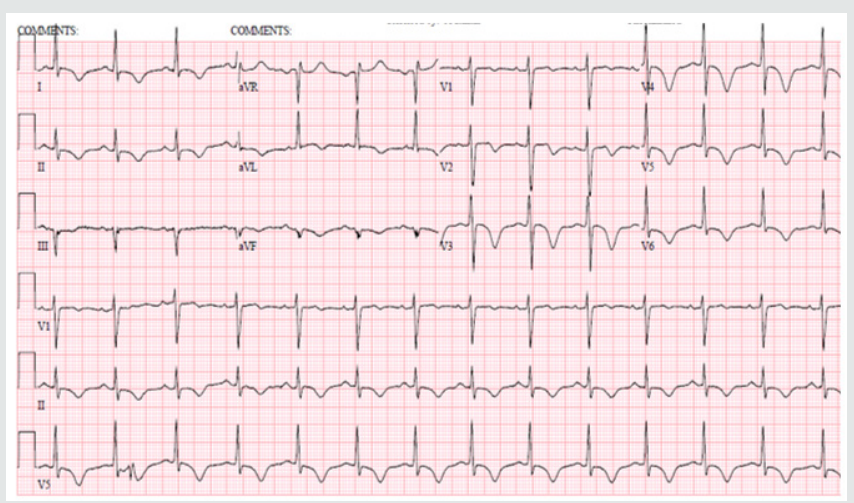

Figure 1: ECG on admission showing sinus rhythm with deep T-wave inversions in both precordial and limb leads. 


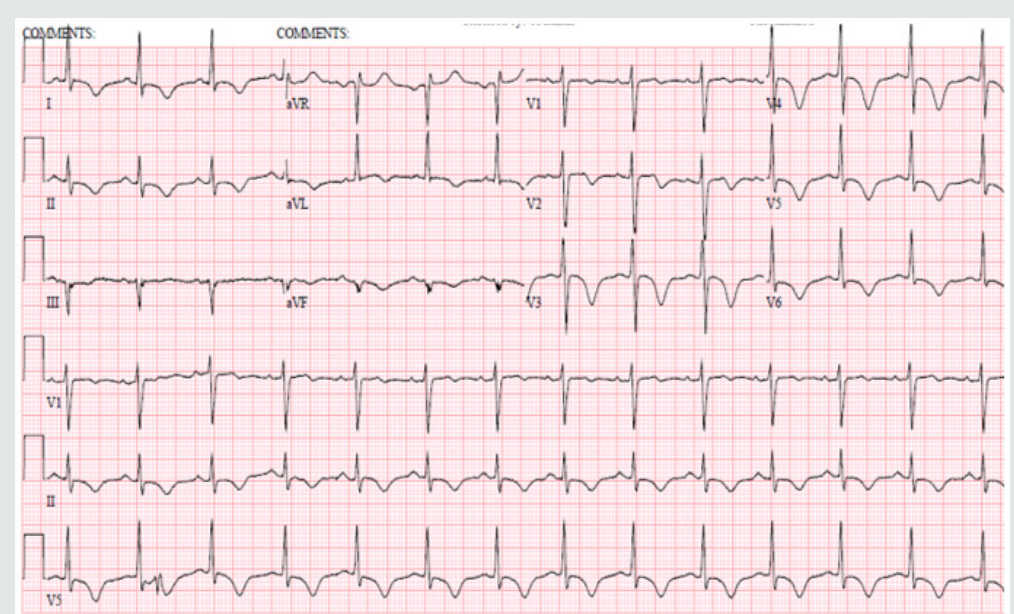

Figure 2: 2-D Transthoracic echocardiogram (apical view) revealing typical apical ballooning with akinetic apical wall and preserved basal contraction of left ventricle.

Interestingly, her inpatient coronary angiogram revealed severe triple vessel coronary artery disease (CAD). This was evident by the presence of mild disease in the left main stem, severe mid vessel calcific LAD lesion, severe obtuse marginal (OM1) disease (Figure 3) and chronic total occlusion of her mid right coronary artery (Figures 4a \& 4b). However, her distal RCA appears well collateralized from contralateral LC system. The left ventriculogram revealed moderately impaired systolic function with moderate mid to apical anterior and inferior wall hypokinesis, sparing the basal segments consistent with "apical ballooning". Figure 5, LV in systole] Given her clinical presentation (albeit coronary artery disease and LV dysfunction), multidisciplinary consensus favored medical management as NSTEMI. Expectedly patient agreed and preferred this option too. She was discharged on appropriate treatment and secondary prevention. Interestingly, her Cardiac MRI (CMR) scan at 1-month post discharge showed near normalization of LV function with calculated EF 50\% with no evidence of infarction or myocardial oedema or fibrosis. Thence, improvement in her LV function with no evidence of infarction on CMR confirms the diagnosis of Takotsubo Cardiomyopathy, despite her significant CAD. At 12-month follow up, patient still remains stable and symptom free; therefore, with plans to consider surgical coronary revascularization, if worsening symptoms on top of medical therapy [2,3].

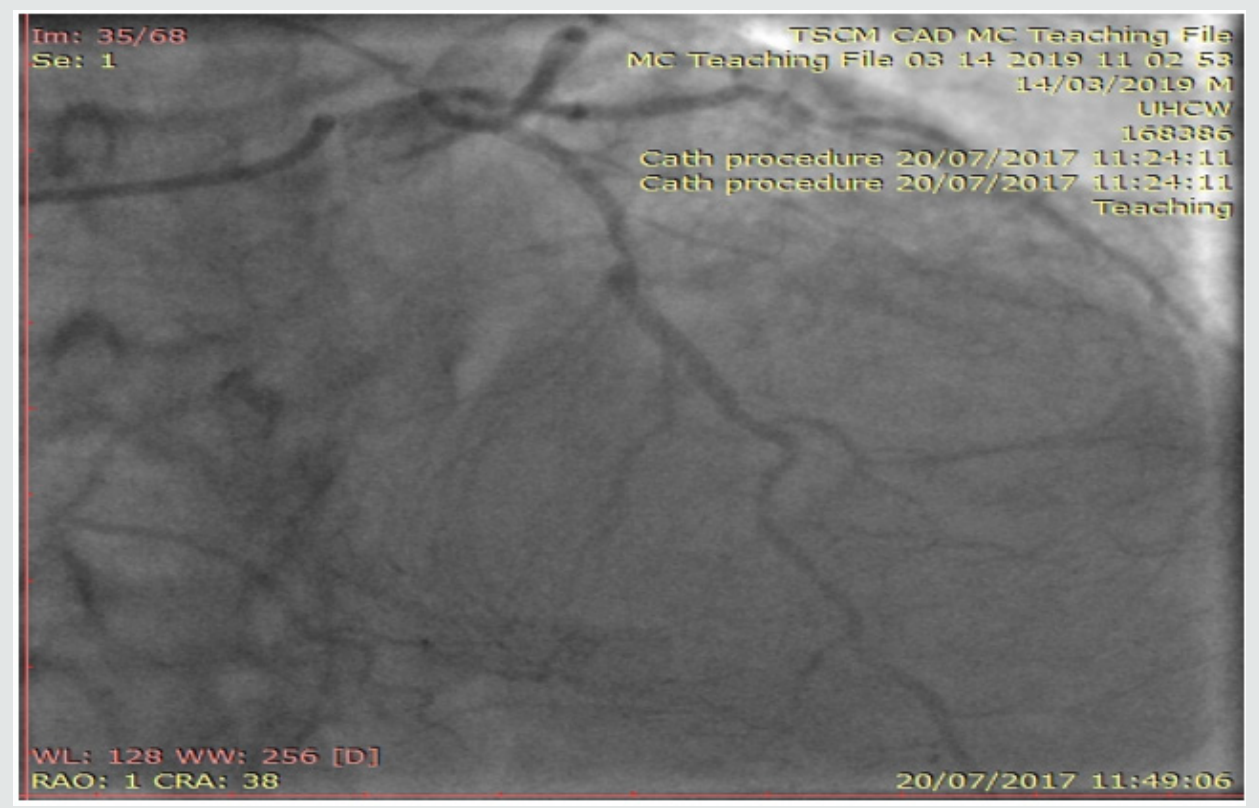

Figure 3: Coronary angiogram of left system revealing severe mid calcific left anterior descending artery disease with contralateral collaterals to distal right coronary artery. 


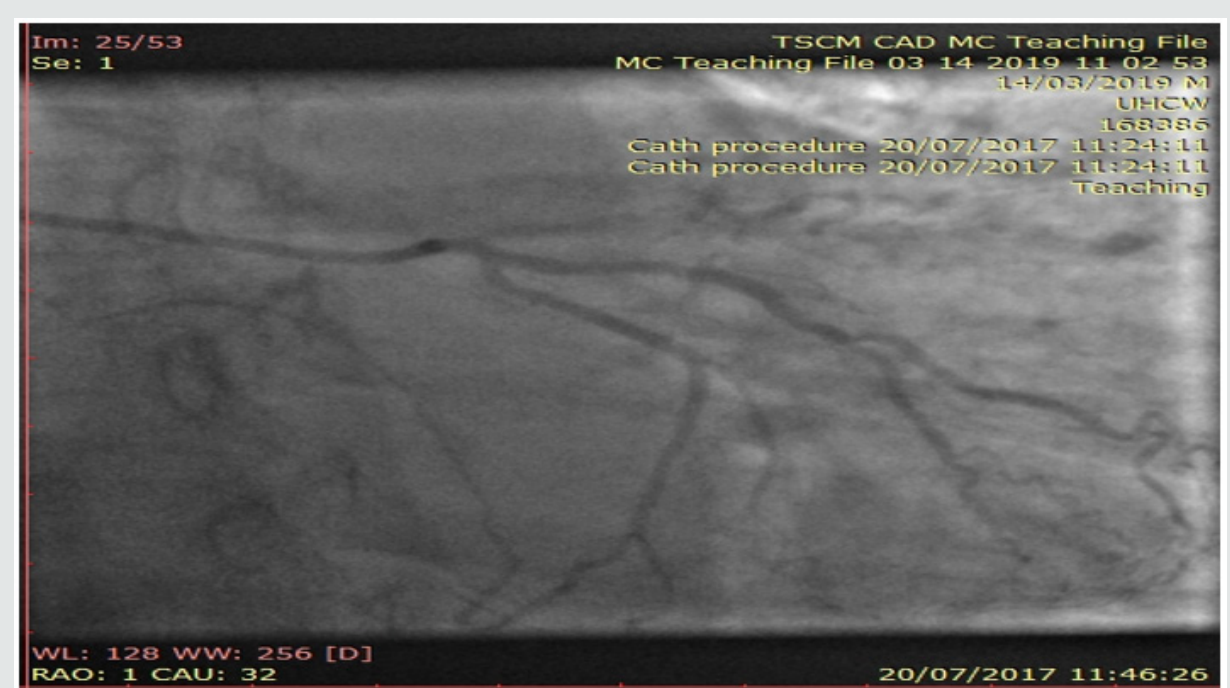

Figure 4a: Coronary angiogram of left system showing severe branch vessel disease in obtuse marginal branch of left circumflex artery.

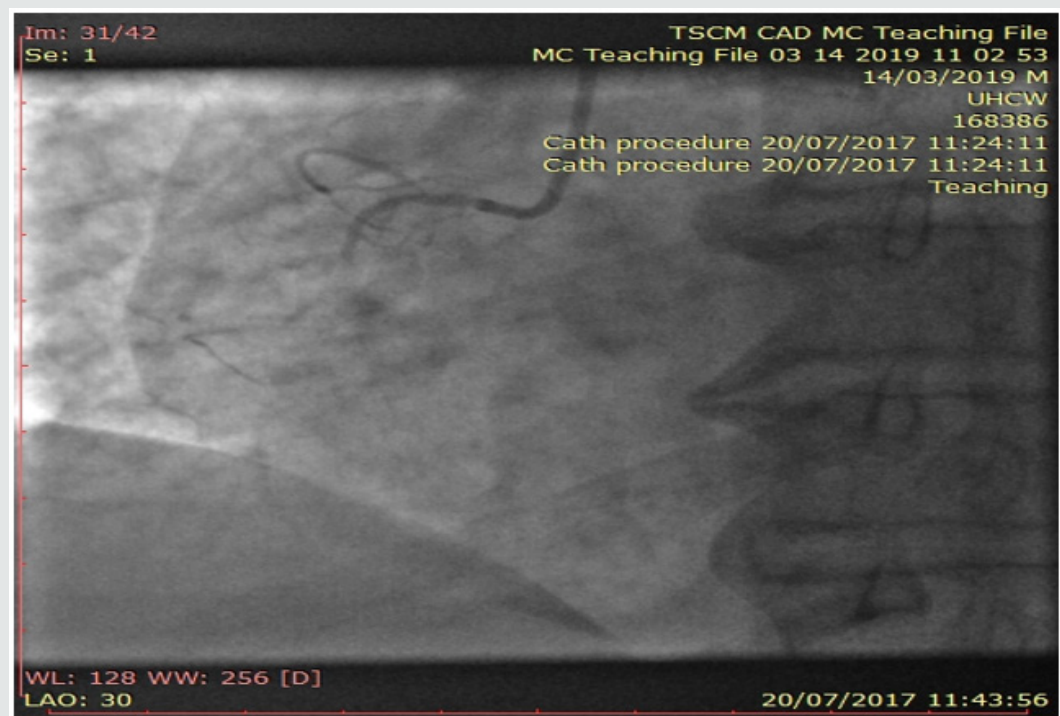

Figure 4b: Angiogram of right coronary artery showing chronic total occlusion at the proximal segment.

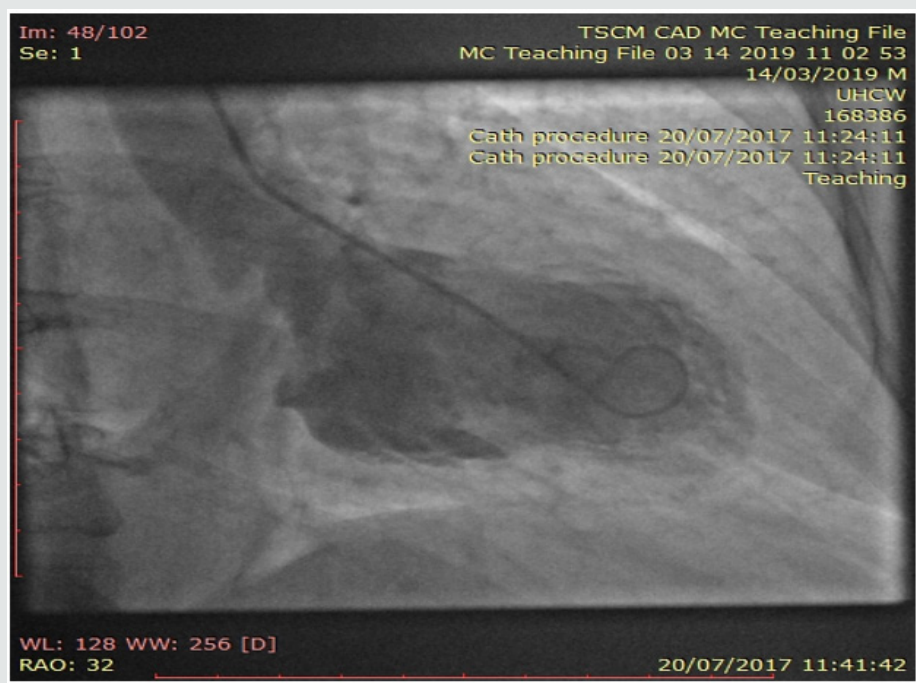

Figure 5: Left ventriculogram at systole confirming the typical pattern of "apical ballooning" sparring the basal anterior and inferior segments. 


\section{Discussion}

With her established macrovascular risk factors for CAD (hypertension and age) and clinical presentation together with abnormalities in ECG, cardiac enzymes and echocardiogram, the working differential diagnoses were:

a. Acute Coronary Syndrome (ACS) i.e., Non-ST elevation myocardial infarction

b. TakotSubo Cardiomyopathy or transient apical ballooning syndrome or stress induced cardiomyopathy or broken heart syndrome.

Conventional electrocardiogram (ECG) still remains a costeffective important tool in the assessment of CAD. Nevertheless, its low sensitivity $(<50 \%)$ and specificity $(<80 \%)$ in predicting coronary disease still remains a major limitation. Clinicians mostly rely on surface ECGs to localize ischaemic myocardial territory (highly specific) prior to coronary revascularization of the culprit vessel(s) routinely in ACS patients. Clinically TSCM (typical LV appearance of Japanese "Octopus trap" with apical ballooning) often mimics AMI (chest pains, elevated cardiac enzymes and ECG changes) and is characterized by a transient left ventricular dysfunction. Though its incidence and prevalence doesn't appear to have vastly changed over the years; however clinicians are more comfortable in diagnosing TSCM with better access to imaging (echocardiogram, Cardiac MRI) and invasive investigations (coronary angiogram) nowadays. The Revised Mayo Clinic Criteria suggested the presence of the following to confirm the diagnosis of TSCM $[4,5]$.

a. Transient hypokinesis, akinesis or dyskinesis of the left ventricular mid segments with or without apical involvementdistinctively the extent of regional wall motion abnormalities observed will be beyond the distribution of single epicardial coronary vessel and with often a stressful trigger (but not always present).

b. Absence of obstructive coronary artery disease or angiographic evidence of plaque rupture

c. New electrocardiographic abnormalities or modest elevation in cardiac Troponin

d. Absence of pheochromocytoma and myocarditis.

However, concomitant coronary artery disease (CAD) has been reported in $10-29 \%$ of patients who have been diagnosed with TSCM. In the International Takotsubo Registry $(n=1750)$ $15.3 \%$ had some degree of coronary artery disease, although the extent of coronary artery involvement was not discussed in TSCM patients. The new Inter TAK diagnostic criteria highlight the clinical entity that TSCM can exist with concomitant CAD too (Table 1). Our patient meets with these diagnostic criteria for "Takotsubo Cardiomyopathy" together confirming that there was no evidence of infarction in her Cardiac MRI (no sub-endocardial late gadolinium enhancement in her LV or microvascular oedema) as well as complete recovery of her LV function rather than the initially presumed diagnosis of ACS [6].

Table 1: International Takotsubo Diagnostic Criteria (8).

\begin{tabular}{|c|c|}
\hline 1 & $\begin{array}{r}\text { Patients show transient left ventricular dysfunction (hypokinesia, } \\
\text { akinesia or dyskinesia) presenting as apical ballooning or } \\
\text { mid-ventricular, basal or focal wall motion abnormalities. Right } \\
\text { ventricular involvement can be present. }\end{array}$ \\
\hline 2 & $\begin{array}{r}\text { An emotional, physical, or combined trigger can precede the } \\
\text { Takotsubo syndrome event, but this is not obligatory }\end{array}$ \\
\hline 3 & $\begin{array}{r}\text { Neurologic disorders (e.g. subarachnoid haemorrhage, } \\
\text { stroke/transient ischaemic attack, or seizures) as well as } \\
\text { pheochromocytoma may serve as triggers for Takotsubo syndrome }\end{array}$ \\
\hline 4 & $\begin{array}{r}\text { New ECG abnormalities are present (ST-segment elevation, ST- } \\
\text { segment depression, T-wave inversion and QTc prolongation); } \\
\text { however, rare cases exist without any ECG changes. }\end{array}$ \\
\hline 5 & $\begin{array}{r}\text { Levels of cardiac biomarkers (troponin and CK) are moderately } \\
\text { elevated in most cases; significant elevation of brain natriuretic } \\
\text { peptide is common }\end{array}$ \\
\hline 6 & $\begin{array}{r}\text { Significant coronary artery disease is not a contraindication in } \\
\text { Takotsubo syndrome }\end{array}$ \\
\hline 7 & Patients have no evidence of infectious mononucleosis \\
\hline
\end{tabular}

The pathophysiology for the condition is not well completely understood yet; nonetheless, stress related acute catecholaminergic surge, multi-vessel epicardial coronary spasm, micro-vascular dysfunction, abnormal fatty acid metabolism, myocardial stunning, micro-infarction, direct catecholamine toxicity to myocytes and possible reperfusion injury were proposed as possible mechanisms. On top of the typical transient apical ballooning, other atypical variants including inverted or reversed, mid ventricular or localized have been reported in the literature. In the majority of cases of suspected TSCM, it is appropriate to perform coronary angiography to exclude an acute coronary syndrome given the overlap of symptoms. Also, there is little evidence to support the clinicians in the management of TSCM with co-existent coronary artery disease as highlighted in this case and the clinical dilemma encountered. Therefore, we believe long term follow-up would be really helpful in understanding the disease as well as their management and prognosis for these TSCM patients $[7,8]$.

\section{Conclusion}

We report an unusual case of Takotsubo Cardiomyopathy with co-existent severe triple vessel coronary artery disease. This case illustrates the importance of good history taking and the judicious use of diagnostic imaging to arrive at a prompt diagnosis and appropriate management. With limited evidence in literature to support clinicians as how best to manage these patients with severe coronary artery disease with Takotsubo Cardiomyopathy, we highlight the need for International registries with long term follow-ups. 


\section{References}

1. Herring N, Paterson DJ (2006) ECG diagnosis of acute ischaemia and infarction: past, present and future. QJM 99(4): 219-230.

2. (2003) Use of the electrocardiogram in acute myocardial infarction. $\mathrm{N}$ Engl J Med 348: 933-940.

3. Sato H (1990) Takotsubo type cardiomyopathy due to multivessel spasm. In: Kodama K, Haze K, Hon M, editors. Clinical aspect of myocardial injury: from ischemia to heart failure. Kagaku Hyoronsha 56-64.

4. Scantlebury D, Prasad A (2014) Diagnosis of Takotsubo Cardiomyopathy - Mayo Clinic Criteria. Circulation Journal 78(9): 2129-2139.

5. Ghadri JR, Wittstein IS, Prasad A, Sharkey S, Dote K, et al. (2018) International Expert Consensus Document on Takotsubo Syndrome (Part
I): Clinical Characteristics, Diagnostic Criteria, and Pathophysiology. European Heart Journal 39(22) :2032-2046.

6. Templin C, Ghadri JR, Diekmann J, Napp LC, Bataiosu DR, et al. (2015) Clinical Features and Outcomes of Takotsubo (Stress) Cardiomyopathy. N Engl J Med 373: 929-938.

7. Afonso L, Bachour K, Awad K, Sandidge G (2008) Takotsubo cardiomyopathy: pathogenetic insights and myocardial perfusion kinetics using myocardial contrast echocardiography. Eur J Echocardiogr 9(6): 849-854.

8. Kurisu S, Kihara Y (2016) Editorial: Variants of Takotsubo cardiomyopathy. J Cardiol Cases 14(1): 24-25.

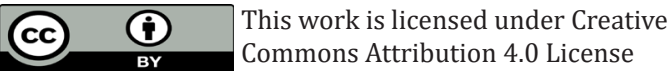

To Submit Your Article Click Here: Submit Article

DOI: 10.32474/ACR.2019.02.000132

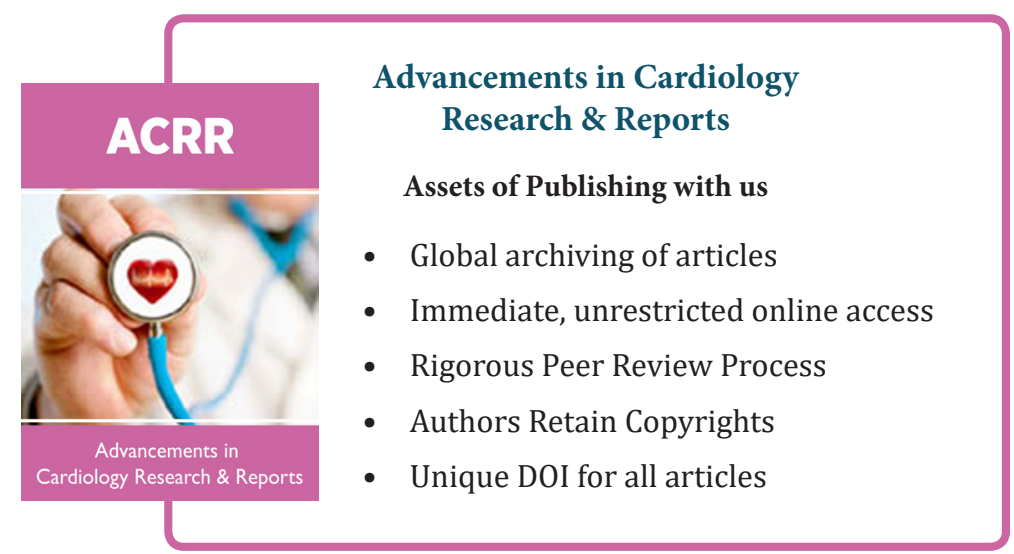

